\title{
Criminologie
}

\section{Transformations dans la santé mentale : entre la science et le sujet}

\section{Transformations in the Mental Health Sector: Between the \\ Science and the Subject \\ Transformaciones de la salud mental: Entre la ciencia y el sujeto}

\section{Yves Cartuyvels}

Volume 48, numéro 1, printemps 2015

Justice et santé mentale

URI : https://id.erudit.org/iderudit/1029346ar

DOI : https://doi.org/10.7202/1029346ar

Aller au sommaire du numéro

Éditeur(s)

Les Presses de l’Université de Montréal

ISSN

0316-0041 (imprimé)

1492-1367 (numérique)

Découvrir la revue

Citer cet article

Cartuyvels, Y. (2015). Transformations dans la santé mentale : entre la science et le sujet. Criminologie, 48(1), 15-35. https://doi.org/10.7202/1029346ar
Résumé de l'article

L'article étudie les transformations historiques dans le domaine de la santé mentale, principalement à travers ses résonances contemporaines dans l'Europe francophone, et plus principalement en France et en Belgique. Il décrit les mutations d'un point de vue institutionnel et matériel en examinant, plus particulièrement et de manière critique, la portée du DSM, l'imaginaire qu'il véhicule et ses conséquences sociales. In fine, ces transformations peuvent être saisies entre une approche scientifique qui rêverait de se débarrasser du sujet humain et une autre qui le tient pour essentiel et irréductible. 


\title{
Transformations dans la santé mentale: entre la science et le sujet
}

\author{
Yves Cartuyvels ${ }^{1}$ \\ Professeur \\ Université Saint-Louis-Bruxelles \\ yves.cartuyvels@usaintlouis.be
}

RÉSUMÉ - L'article étudie les transformations historiques dans le domaine de la santé mentale, principalement à travers ses résonances contemporaines dans l'Europe francophone, et plus principalement en France et en Belgique. Il décrit les mutations d'un point de vue institutionnel et matériel en examinant, plus particulièrement et de manière critique, la portée du DSM, l'imaginaire qu'il véhicule et ses conséquences sociales. In fine, ces transformations peuvent être saisies entre une approche scientifique qui rêverait de se débarrasser du sujet humain et une autre qui le tient pour essentiel et irréductible.

MOTS CLÉS - Santé mentale, transformations historiques, évolution, DSM, critique.

\section{Introduction}

Depuis l'époque moderne, l'histoire de la santé mentale peut se lire selon une logique marquée par des éléments de continuité et de ruptures. Associée d'abord à une pathologie de la liberté, la folie donnera naissance à l'idéal d'un traitement moral qui ouvre la voie à une approche psychopathologique de l'aliénation et de ses diverses manifestations, au cœur de la structure asilaire. Cette perspective mentaliste sera cependant mise en question par une approche organiciste, marquée par un rêve classificateur fondé sur l'observation et la catégorisation. Sur fond de cette opposition, un certain nombre de transformations vont marquer le champ de la santé mentale par la suite. Sur le plan

1. Université Saint-Louis-Bruxelles, 43, boulevard du Jardin Botanique, 1000 Bruxelles. 
institutionnel, l'hôpital succède à l'asile, avant de donner lieu à des modes d'interventions ambulatoires complémentaires, dans un mouvement qui souligne à la fois la dimension de santé publique de la problématique et son jaillissement hors des frontières du secteur santé. Sur le plan matériel, la notion de maladie mentale cède la place à celle de trouble mental, dont l'apparition témoigne d'une extension de la souffrance psychique, d'une nouvelle lecture de celle-ci en termes de handicap ou de déficit et d'un recours accru au pharmakon comme modalité d'accompagnement d'un usager de soins. Émerge de manière corrélative une nouvelle lecture descriptive et pragmatique du trouble mental, popularisée par l'influence du Diagnostic and Statistical Manual of Mental Disorders (DSM).

\section{Mutations contemporaines de la santé mentale: sous le signe de l'extension}

L'extension institutionnelle: de l'hôpital à l'ambulatoire, du médecin au sujet responsable comme vecteur de soins

$\mathrm{Au} \mathrm{xx}^{\mathrm{e}}$ siècle, l'hôpital succède à l'asile, sans éliminer ce dernier. Porteur dans le monde de la santé de la logique des droits-créances propre au projet de l'État social, l'hôpital se pose en pivot d'un service de santé repris en main par l'État et octroyé à des assurés sociaux dont il s'agit de respecter le droit aux soins et la dignité (Schweyer, 2006, p. 48). L'interventionnisme redistributeur du projet de l'État providence fonctionne sur une logique de secteur, par le recours à des systèmes cloisonnés prenant appui sur des acteurs institutionnels puissants susceptibles d'organiser de manière rationnelle le droit aux droits. Dans le secteur santé, l'hôpital est cet acteur qui permet à l'État d'imposer sa marque sur les politiques de santé. L'hôpital se positionne rapidement au cœur des politiques de santé mentale, contribuant à transformer progressivement la question de la folie sous ses diverses formes reconnues en problème de santé publique. Comme en matière de déviance, la logique de contrôle social se double d'un projet de réintégration, ce qui suppose que la guérison $\mathrm{du}$ «malade» apparaisse comme une visée possible de l'intervention hospitalière confiée à des professionnels (Genard et Donnay, 2002, p. 73). Aux confins d'une intervention psychothérapeutique et du recours aux moyens techniques de la pharmacopée, 
la médicalisation de la santé mentale se précise en régime expert: pas moins que dans le modèle asilaire, le médecin psychiatre est au cœur du modèle hospitalier. C'est lui qui est le détenteur du savoir, c'est lui qui détient les clés du traitement, qu'il s'agisse de recourir à la psychothérapie ou d'imposer une médication à un patient oscillant entre sujet et objet de soins.

Le modèle hospitalier dominera jusque dans les années 1970. Par la suite, sa logique d'intervention va s'étendre, se diffuser et partiellement se diluer au-delà de sa sphère traditionnelle d'intervention dans le cadre d'un changement général des modes d'action publique. Dans les années 1980, une critique se déploie sur le fonctionnement bureaucratique de l'État social et ses rationalités systémiques et programmatrices, que l'on juge aveugles et déconnectées du «monde vécu» (Habermas, 1988) des citoyens. Elle donne naissance à l'émergence «d'un État animateur» (Donzelot et Estebe, 1994) qui se fait promoteur et partenaire d'une multiactorial governance (Crawford, 2001) au cœur d'une «société en réseau» (Castells, 1996). Dans le champ de la santé mentale, l'irruption de «l'État réseau» (Genard et Donnay, 2002, p. 3) a plusieurs conséquences.

Tout d'abord, on assiste à une extension de la sphère d'intervention des services et des soins au-delà des frontières traditionnelles du «secteur» de la santé mentale. Tant dans le monde du travail (Périlleux, 1996) que dans celui de la justice (De Coninck et al., 2005) par exemple, se mettent en place des modes d'intervention partenariale qui dépassent les cloisonnements antérieurs et traduisent une dé-différentiation des champs. Les frontières de la santé mentale se brouillent, en réponse aussi à de nouvelles demandes qui explosent à ses portes et qui accentuent la perception de la santé mentale comme problématique de santé publique (cf. infra). Ensuite, l'accent est mis, comme dans d'autres domaines de l'action publique, sur une intervention dans le milieu du patient. Cela se traduit par la promotion de services ambulatoires, appelés à relayer ou remplacer l'hospitalisation psychiatrique (Vrancken, 2002, p. 39). Émerge, dans ce cadre, une myriade de nouveaux professionnels qui s'intègrent dans des «réseaux de soins» fonctionnant selon une logique connexionniste et non plus seulement sectorielle et linéaire. Enfin, la logique participative de l'État réseau accroît l'implication de l'«usager» dans l'élaboration des politiques de soins. Plateformes de concertation et associations de patients se développent, favorisant la construction de savoirs hybrides (Callon, Lascoumes et Barthes, 2001) 
qui viennent concurrencer et parfois remettre en question la prédominance du savoir expert ${ }^{2}$. Cet accent mis sur l'usager reste néanmoins ambivalent: oscillant entre l'idéal d'un sujet réflexif et le «démocratisme ${ }^{3} »$, ce déplacement vers le sujet comme source de savoir (et de prise en charge) traduit aussi les accents néo-libéraux d'une culture managériale de l'autonomisation et de la responsabilisation. L'idéal d'activation, associé au modèle d'un sujet entrepreneur de soi tel que le promeut le discours de l'«État social actif» (Dumont, 2008), s'infiltre dans le domaine de la santé mentale comme dans les autres domaines de l'action publique. Il est bien intériorisé, comme en témoigne le succès croissant d'une littérature scientifique sur le développement personnel (Marquis, 2013) qui répond à un principe d'autorésilience. Mais cette intériorisation des nouveaux impératifs du management traduit aussi une évolution dans les modes de subjectivation qui n'est pas sans incidence sur l'extension des demandes et leurs contours dans le champ de la santé mentale. Elle explique pour partie l'extension du champ de la santé mentale à de nouvelles pathologies ou nouveaux troubles de l'existence.

\section{L'extension et la diversification du malaise dans la civilisation: de la maladie mentale aux troubles de l'existence}

À la dé-différentiation du champ de la santé mentale et à l'extension de l'offre hors de ses frontières traditionnelles correspond une prolifération de la demande. Certes, la psychiatrie reste concernée par les pathologies lourdes traitées à l'asile où à l'hôpital. Mais son champ partenarial d'intervention s'est étendu à une multitude d'autres pathologies, au-delà des frontières traditionnelles de la folie ou de la psychose (Ehrenberg et Lovell, 2001, p. 13-16). Les murs de l'asile sont tombés mais, parallèlement, un ensemble de souffrances s'est progressivement mis à sourdre de partout. Elles trouvent une réponse dans la santé mentale (Ehrenberg, 2004, p. 133). Si on ajoute à cette liste la multiplication des «états limites» et des «doubles diagnostics», l'explosion du repérage de l'hyperactivité chez les enfants, l'identification croissante de «troubles de la personnalité» ou, dans un autre registre, celle

2. Le rôle joué par les associations de parents d'enfants autistes en France donne un bon exemple de cette évolution.

3. L'expression est de Lebrun (2007), cité dans Adam (2012). 
de troubles alimentaires, on trouve une liste «assez hétéroclite» de nouvelles pathologies (Demailly, 2011, p. 43).

L'interprétation de ces nouveaux symptômes, dont Freud (1995) ferait sans doute aujourd'hui l'expression du «malaise dans la civilisation» contemporain, peut bien évidemment donner lieu à des interprétations différentes qui, en l'espèce, sont sans doute plus complémentaires que contradictoires. Une première grille de lecture souligne une psychiatrisation de l'existence qui n'a d'autre effet que de médicaliser des problèmes avant tout politiques et sociaux. Psychiatriser revient ici à individualiser le trouble et à en dénier la dimension sociale et collective plus fondamentale ${ }^{4}$. La lecture psychique de problèmes sociaux jouerait un rôle de diversion: elle détournerait le regard des vrais enjeux, à savoir la violence du monde social (source de précarisation) ou encore la montée de l'intolérance sociale à des comportements dont la lecture comme déviance est le fruit d'une construction sociale.

Une seconde approche renvoie aux réalités de la «métamorphose de la question sociale» (Castel, 1995) et aux effets psychiques induits par «La Misère du monde» (Bourdieu, 1993). Dans des sociétés postindustrielles marquées par une tertiarisation de l'économie, la souffrance psychique tend réellement à remplacer la souffrance corporelle comme lieu d'expression de la misère sociale. Une lecture psychique prend dès lors légitimement la place d'une lecture physique de la violence sociale (Vrancken, 2002, p. 40). En outre, dans ces sociétés qui sont aussi caractérisées par la précarisation de nombre de trajectoires individuelles, la souffrance psychique se lit comme la traduction subjective des effets de «l'insécurité sociale» (Castel, 2003). Souffrance psychique et désaffiliation sociale se superposent sur fond de problématiques croisées, ce dont témoignent nombre d'intervenants de terrain ${ }^{5}$. Et si le symptôme appelle ici un traitement individuel, il n'en garde pas moins un statut de témoin de la question sociale ${ }^{6}$.

4. Demailly évoque ici des auteurs comme Pignarre, Muccielli, Fassin ou Castel (Demailly, 2011, p. 44).

5. Pour une illustration dans le champ de la toxicomanie, comme illustration d'une pathologie individuelle sur fond de déshérence sociale, voir Cartuyvels (2004).

6. Une troisième approche met l'accent sur les transformations de la subjectivité qu'induisent les mutations du régime symbolique propre à la société contemporaine. Voir, entre autres, Erhenberg (1991, 2000); Lebrun (2007); Legendre (2003). 
Quelle qu'en soit l'explication, le résultat de cette évolution est une reconfiguration profonde du champ des «troubles mentaux», dont la surface est largement indéterminée et le périmètre extensible à l'infini. Comme le souligne Demailly (2011) pour la société française, autour des images de la déviance (troubles sexuels, comportements "antisociaux», dépendances criminalisées), de la souffrance (dépression et autres phénomènes répertoriés d'angoisse ou d'anxiété) et du déficit (psychose, autisme), émerge une nouvelle représentation sociale de la pathologie mentale (Demailly, 2011, p. 55). Cette évolution en amènerait une autre, qui peut se décliner autour d'un triple enjeu: la sortie de l'univers binaire santé-pathologie; le remplacement de l'imaginaire de la guérison par celui de l'accompagnement; et, corollairement, la normalisation du médicament comme soutien dans la durée à une souffrance qui se chronicise.

L'extension du recours au pharmakon: de la guérison du malade à l'accompagnement d'un usager chronique

L'extension de la maladie mentale à la souffrance psychique sous ses formes multiples a pour effet premier de diluer un peu plus l'imaginaire binaire du normal et du pathologique, tout comme la frontière entre problème psychique et problème physique. Avec le «trouble» émerge un déplacement sémantique significatif qui fait glisser le malaise du côté du handicap ou du déficit en regard d'une norme de socialisation: «aujourd'hui, le registre du déficit, brouillant à nouveau les frontières du handicap mental et du handicap physique, supplante petit à petit celui de la «maladie»» (Demailly, 2011, p. 35). Il s'agit dès lors moins de "guérir» un malade, au sens médical du terme qui consiste à supprimer un symptôme au point d'en arrêter le traitement, que d'accompagner un usager à mieux vivre avec le handicap que produit une souffrance psychique chronique. Laccompagnement devient un nouvel impératif qui répond à des souffrances ou troubles psychiques multiples dont il est moins important d'interroger le nœud que de mesurer et contrôler l'impact sur la vie quotidienne (Ehrenberg, 2004, p. 148-149). Avec le glissement de la maladie au trouble, c'est un idéal de capacitation du sujet - lequel répond bien aux impératifs d'autonomisation et de responsabilisation du discours social contemporain - qui se glisse au sein d'un champ de la santé mentale en extension pour répondre aux préoccupations de la socialisation comme 
du contrôle social. En Belgique, les débats des années 1990 sur le recours à la méthadone comme drogue de substitution dans le champ de la toxicomanie ont bien illustré cette évolution : avec la méthadone, qualifiée tantôt de "sanitaire», tantôt de «sécuritaire», il ne s'agissait plus de viser un idéal d'abstinence synonyme de guérison mais bien d'accompagner à long terme un usager de drogues avec son handicap dans la vie sociale (Bauherz, Kaminski et Zombek, 1992). La santé mentale s'inscrit ici dans une perspective de gouvernementalité où il s'agit de penser des dispositifs de gestion d'individus que le trouble rend moins fonctionnels pour eux-mêmes comme pour la société (Demailly, 2011, p. 37).

Dans cette perspective, le recours au médicament comme support chimique se banalise. Comme le souligne un psychiatre, on peut certes regretter les défauts des molécules, dénoncer les stratégies des firmes pharmaceutiques, on ne peut plus se passer aujourd'hui dans la pratique psychiatrique du recours aux neuroleptiques (Feys, 2009, p. 243). Dès lors que la question est moins de guérir que d'accompagner, de supprimer un symptôme que d'atténuer les effets d'un trouble ou de le supprimer, le recours au médicament s'impose comme outil de stabilisation à long terme à des troubles dont la chronicité devient la règle. Objet qui permet de renforcer les capacités individuelles ou d'atténuer les souffrances, le médicament permet de lutter contre les déficits existentiels, de prévenir les rechutes et d'éviter des processus de désocialisation. Dans le domaine de l'internement en Belgique par exemple, la compliance au traitement médicamenteux est perçue comme une garantie à ce triple point de vue et c'est bien ce qui explique qu'elle soit érigée en critère décisif d'octroi d'une libération à l'essai (Cartuyvels, 2012).

L'explosion du recours au médicament est par ailleurs associée au succès de la nouvelle approche syndromique de la maladie mentale qu'incarne la «bible» de la psychiatrie américaine et mondiale, le Diagnostic and Statistical Manual of Mental Disorders ou DSM. Depuis sa troisième version publiée en 1980, le DSM propose un découpage descriptif de la maladie mentale en troubles toujours plus spécifiques auquel répond une «inflation» de prescriptions médicamenteuses, il est vrai encouragée par le marketing pharmaceutique même quand cette prescription n'apparaît pas ou plus nécessaire (Frances, 2013a). 


\section{La logique DSM au cœur de la santé mentale: le rêve d'une science sans le sujet}

\section{La logique du DSM : pragmatisme athéorique et sémiologie descriptive}

La publication du Diagnostic and Statistical Manual of Mental Disorders III en 1980 aux États-Unis constitue un tournant incontestable dans la culture du diagnostic et du traitement. Succédant au DSM-II (1962), qui était lui-même une révision du DSM-I (1952), le DSM-III s'inscrit dans la grande logique classificatoire incarnée par la figure de Kraepelin au tournant des $\mathrm{xIX}^{\mathrm{e}}$ et $\mathrm{Xx}^{\mathrm{e}}$ siècles. Sa construction traduit la victoire des «néo-kraepeliens» sur les «psychanalystes» dont l'approche étiologique avait dominé la rédaction du DSM-II (Adam, 2012, p. 141). Légèrement modifié par le DSM-III-R (1987), le manuel sera à son tour révisé par le DSM-IV (1994), lui-même modifié par le DSM-IV-TR, publié en 2000. Enfin, une nouvelle version censée incarner un «tournant paradigmatique», le DSM-5, a été publiée en mai 2013.

Au départ, le DSM-III se présente comme un projet «athéorique» et dégagé de présupposés moraux dont la visée est essentiellement pragmatique en réponse au problème de la fiabilité de l'expertise psychiatrique. L'absence de consensus dans le diagnostic était source de problèmes multiples: d'une part, les désaccords entre psychiatres en fonction de leur orientation théorique conduisaient à des diagnostics dissonants et à des traitements diversifiés, ce qui constituait une menace pour l'autorité de la discipline. D'autre part, l'absence de consensus représentait un problème pour la recherche, financée pour une grande part par l'industrie pharmaceutique. À partir des années 1980, le DSM, comme c'est rappelé dans l'introduction du DSM-IV-TR, vise donc à «fournir un guide utile aux cliniciens», à «faciliter la recherche» et à «améliorer la communication entre chercheurs et cliniciens» (American Psychiatric Association [APA], 2002). Le défi majeur est celui de la création d'un outil statistique classificateur uniforme permettant des diagnostics réplicables grâce à l'adoption d'un outil de mesure commun qui mette fin aux divergences entre experts. Autrement dit, l'enjeu est celui de la fidélité du diagnostic: il s'agit que, confrontés à une même pathologie, des psychiatres issus de cultures et d'horizons divers, utilisant une même grille d'indicateurs, aboutissent à un diagnostic semblable et puissent proposer le traitement spécifique adéquat associé au «trouble» repéré. 
Pour ce faire, les auteurs du DSM-III vont faire le choix d'une sémiologie «cardinale ${ }^{7} »$, fondée sur la seule description de troubles, assortis de critères identificatoires dont la réunion permet d'attester l'existence du trouble. Le recours à une classification multiaxiale - le DSM-III s'articule autour de cinq axes - s'apparente à une logique de dossier et vise «à recueillir sur un patient des données aussi complètes que possible afin de permettre à terme une appréhension statistique des coordonnées des troubles mentaux» (Maleval, 2003, p. 43). Le choix descriptif, qui met l'accent sur l'existence de signes objectifs aisément repérables faisant l'objet d'un consensus entre psychiatres, fait l'impasse sur l'étiologie de la maladie: sauf là où elle est clairement établie, toute spéculation sur les causes est éliminée, ce qui atteste du caractère «révolutionnaire» de l'entreprise (APA, 1983, IX). Cette élimination se justifie d'une part, parce qu'elle renvoie à des débats indécidables sur l'essence de la maladie; d'autre part, parce que l'«athéorisme» descriptif est la condition de l'acceptation et de l'utilisation du manuel par tous (Chapireau, 2013, p. 72).

\section{Les critiques du DSM: d'une neutralité axiologique utopique aux effets sociaux d'un instrument normatif}

Dans le monde francophone européen, plusieurs critiques seront formulées à l'égard de la logique qu'inaugure le DSM-III et que poursuivent ses versions ultérieures. S'inspirant pour une large part du débat anglo-saxon ${ }^{8}$, ces critiques ont trouvé un nouvel élan à la sortie du DSM-5 dans des pays (la France et la Belgique) où le poids de la psychanalyse explique en bonne partie les résistances à ce type d'instrument ${ }^{9}$. La neutralité axiologique de l'instrument est ainsi mise en cause.

7. Feys (2009) souligne qu'en sémiologie médicale, «on parle de signes cardinaux lorsque plusieurs signes doivent être ensemble présents pour que l'on puisse poser un diagnostic (...). Dans le cardinalat, chaque signe pris à part est polysémique mais leur réunion est monosémique. C'est la réunion qui permet le diagnostic» (p. 112).

8. Sur ce débat, on lira, entre autres, Aragona (2009); Cooper (2005); Mayes et Horwitz (2005); Paris et Phillips (2013); Wakefield (1992).

9. Si le DSM reste peu utilisé en France, cela ne signifie pas que son influence soit négligeable. D'une part, la terminologie issue du DSM est largement utilisée dans les manuels de psychiatrie (Fineltain, 2011, p. 11) et le «langage» DSM a bien pénétré les pratiques psychiatriques. D'autre part, une institution officielle comme l'Institut national de la santé et de la recherche médicale [Inserm] revendique le recours à des échelles classificatrices neutres et objectives comme le CIM-9 ou le DSM (Inserm, 2005, p. 5). Cela explique que le débat sur le DSM ait bien lieu en France, comme l'illustre l'initiative Stop DSM (www.Stop-DSM.org) ainsi que des publications récentes sur cet instrument (Demazeux, 2013). 
Un second type de critique a trait aux effets sociaux du néopositivisme incarné par le DSM. D'instrument de communication athéorique et consensuel au départ, son usage obligatoire et les catégories normatives implicites qui tissent sa configuration des troubles et des critères font de cet instrument «soucieux de normalité plus que de santé... un outil planétaire de contrôle social» (Martens, 2002, p. 48). Certes, comme le soulignent les auteurs de l'introduction du DSM-IV-TR, l'instrument ne s'intéresse au trouble que dans la mesure «où il est considéré comme la manifestation d'un dysfonctionnement comportemental, psychologique ou biologique de l'individu». Il n'est donc, pour ces derniers, pas marqué normativement. D'ailleurs, «ni un comportement déviant (p. ex.: politique, religieux ou sexuel), ni des conflits existant essentiellement entre l'individu et la société ne sont des troubles mentaux, sauf si la déviance ou le conflit est le symptôme d'un dysfonctionnement chez l'individu concerné» (APA, 2002, XXXV-XXXVI). Mais comment ne pas voir que l'identification du «trouble objectif» repose sur des critères qui renvoient forcément aussi à des normes sociales? Comment ignorer que la définition du trouble a également des effets sociaux en termes d'identification et de stigmatisation? Comme le souligne, dans sa critique virulente du DSM-5, Frances (2010c), un des pères fondateurs du DSM-IV, en l'absence d'une définition valide du trouble mental par la science, le renvoi normatif est inévitable et ouvre la voie à des choix normatifs qui n'ont rien d'«évident». Ainsi, explique Frances (2010c), quels que soient les fondements trouvés sur la base de la littérature scientifique, «je continue à ne trouver aucune raison d'étiqueter comme trouble mental des besoins sexuels, des fantasmes ou des comportements qui sont sans dommage pour autrui et ne causent ni détresse ni dommage à l'individu». Le problème, explique encore l'auteur, est que ce genre de question est généralement éliminé par les experts au motif que «leur responsabilité s'arrête à la science» (Frances, 2013a) ${ }^{10}$. Or, comme le souligne Ehrenberg (2006), dès lors que la science intervient sur des faits de société, «elle se trouve automatiquement prise dans les conflits que ces questions occasionnent toujours», parce qu'il s'agit de «faits sociaux» qui sont toujours aussi des «faits de valeur» (p. 90). Vouloir ne rien en

10. Un constat similaire peut être fait à propos des experts mobilisés par l'Inserm en France dans leur rapport publié en 2005 sur le Trouble des conduites chez l'enfant et l'adolescent (Cartuyvels, 2009). 
savoir oriente la psychiatrie vers une conception purement instrumentale de la science qui, comme le rappelait Canguilhem à propos de la psychologie béhavioriste, aurait plutôt tendance à l'orienter vers «la préfecture de police» (Canguilhem, 1968, p. 381).

Par ailleurs, la logique multiaxiale du DSM, dénuée d'un principe organisateur théorique, induit une logique d'extension des troubles. La quête de données objectives est infinie et pousse à l'adjonction continue d'axes, de classes et de troubles toujours plus spécifiques, en réponse aussi aux stratégies de conquête de l'industrie pharmaceutique (Lane, 2009). L'inflation des classes diagnostiques est une réalité que ne dément pas l'évolution des diverses versions du DSM. De ce point de vue, le DSM est «le paradigme d'une psychiatrie qui, ayant perdu ses repères et ses fondements, se limite à additionner tous les troubles qui posent problème socialement à un moment ou à un autre. Dès qu'un nouveau comportement apparaît ou dès que la société ne tolère plus un certain type de comportement, le DSM est capable d'objectiver les signes pour en définir le trouble» (Feys, 2009, p. 114). À cet égard, la critique portée de l'intérieur par Frances à l'égard du DSM-5 est sévère. Depuis trois ans, à l'aide de nombreux articles de presse mais aussi dans un ouvrage récent (Frances, 2013b), le psychiatre dénonce les effets d'une logique qui, faisant l'hypothèse d'une continuité entre normal et pathologique, aboutit à «augmenter de manière radicale le nombre des troubles mentaux» (Frances, 2010a). Cette logique inflatoire, dont Frances reconnaît incidemment l'existence dans le DSM-IV également, connaitrait toutefois un saut qualitatif avec le DSM-5: l'instrument inclurait dans les troubles nombre de comportements qui font partie de la vie ordinaire; de même l'abaissement des seuils du diagnostic contribuerait à potentiellement psychiatriser une partie importante de la population normale (Frances, 2010b). En outre, l'adjonction de multiples troubles traduirait les «sujets de prédilection» de tel ou tel psychiatre bien plus que le résultat d'études rigoureuses (Frances, 2013a). À l'inflation des troubles répertoriés répondrait par ailleurs une inflation des troubles diagnostiqués, augmentant considérablement le nombre de faux positifs: le marketing des firmes pharmaceutiques, toujours à l'affût, serait ici particulièrement efficace à l'égard des médecins généralistes. Or, écrit Frances, aux États-Unis, ce sont ces médecins sans formation psychiatrique qui prescrivent $80 \%$ de la médication au terme d'un diagnostic réalisé en moyenne en sept minutes, avec à la clé «la distribution d'un échantillon gratuit de médicament» (Frances, 2013c). 
Pour justifiée qu'elle soit, la critique de Frances reste néanmoins paradoxale. Focalisé sur le DSM-5, l'auteur semble considérer que la dérive qu'il dénonce constitue une sorte de «passage à la limite», un point où la logique intrinsèque du DSM (qu'il ne remet pas en cause) se retourne en son contraire. S'il reconnaît que certaines dérives ou effets problématiques sont déjà présents dans le DSM-IV, il ne semble pas considérer que les problèmes qu'il soulève sont intrinsèquement liés à la logique classificatoire descriptive choisie et que cette voie est autant celle des DSM III et IV que 5. Le caractère construit des définitions et des classifications des troubles et des critères, les conséquences sociales en termes de médicalisation de l'existence et de labellisation de la déviance, le boulevard ouvert à l'instrumentalisation par l'industrie pharmaceutique ne sont bien évidemment pas le propre du DSM-5. Il s'agit bien d'écueils fondamentalement liés à la logique interne du DSM depuis sa troisième version (Singy et Demazeux, 2013). Reste à savoir, relativement à ce concert de critiques, ce qui explique le succès de l'instrument.

L'imaginaire du DSM : entre rationalité managériale, novlangue et Evidence-Based Mental Medicine

À ce stade, on peut se demander ce que traduit fondamentalement la logique interne du DSM sur le plan épistémologique et social. Après tout, le rêve classificatoire est très ancien en santé mentale... On voudrait souligner ici trois caractéristiques fondamentales de la «logique DSM» qui peuvent, pour partie, expliquer son succès.

La première est que le principe classificateur tel qu'il est construit et utilisé dans le DSM traduit le passage à une médecine industrielle de la santé mentale. Prenant appui sur un logiciel de codification de l'information (Demunck, 2004), l'instrument crée «un lien de correspondance univoque entre le diagnostic et le (s) geste (s) technique (s) correspondant (s)» (Advenier et Kapsambelis, 2013, p. 11). Fondé sur des guidelines, un diagnostic à la chaîne en quête d'automaticité se substitue à une approche individualisée et réfléchie de l'acte artisanal de soins: «Dans le mode d'emploi actuel du DSM, penser n'est qu'une variable parasite faisant perdre du temps à l'évaluateur. À l'heure du coaching, diagnostiquer, c'est cocher» (Martens, 2010, p. 86). La formule peut paraitre sévère; elle n'est pas forcément exagérée. Dans leur introduction au DSM-IV-TR, les auteurs reviennent sur la place faite au jugement cli- 
nique. Certes, cette place n'est pas éliminée mais elle est singulièrement limitée. Il s'agit avant tout de repérer les critères listés et toute l'interaction est centrée sur cet objectif. Dans ce cadre, le médecin a une certaine marge d'interprétation et il pourra éventuellement apprécier l'existence du trouble en l'absence de tous les items en principe requis, si «les symptômes présentés sont persistants et sévères». L'automaticité d'une démarche standardisée n'est pas totale mais la marge de manœuvre du clinicien est étroite et doit le rester. En effet, «une interprétation trop souple ou trop particulière des critères et des conventions du DSM-IV réduit nettement son utilité en tant que moyen de communication» (APA, 2002, XXXVII). Dans un contexte dominé par une rationalité instrumentale en quête d'efficience, cette logique de standardisation présente plusieurs avantages. Outre un idéal de communication parfaite, elle permet, dans une perspective managériale, la comparaison et l'évaluation des actes produits et de leur coût. Elle assure aussi aux prestataires de soins une certaine sécurisation - tant que l'on a suivi le protocole, on est à l'abri -, le revers de la médaille étant d'encourager une certaine déresponsabilisation. De ce point de vue, la logique DSM répond bien aux exigences d'un modèle managérial contemporain, tel qu'il se décline avec des caractéristiques semblables dans d'autres domaines de l'action publique, comme la justice (Garapon, 2008).

La seconde caractéristique est l'ambition du DSM-III de créer une langue unique. En recourant aux instruments de la statistique et au consensus, il s'agit d'élaborer une novlangue à caractère universel qui mette fin au pluralisme des classifications antérieures et aux conflits d'interprétation que ce pluralisme pouvait engendrer (Advenier et Kapsambelis, 2013, p. 17). Le rêve est celui d'une communication sans reste, fondée sur un langage neutre et objectif, qui ne laisse plus guère de place aux écarts de langage d'un savoir construit entre les lignes à partir de la pratique. Difficile ici de ne pas penser au mythe de Babel, ce mythe qui, souligne Ost (2009), renvoie à un fantasme récurrent à travers l'histoire de l'humanité: celui d'une langue commune et parfaite, code universel qui transcende l'écart entre le langage et le réel, évite les pertes et assure l'efficacité dans la communication, répond à la confusion qu'entraîne la multiplicité des langues et l'interprétation qui en résulte (Ost, 2009). L'objectif revendiqué par le DSM est celui d'une communication transparente ou encore l'utopie de la suppression du malentendu, alors que ce dernier est sans doute une condition de l'échange, sinon une nécessité dans les relations humaines (Van 
Campenhoudt, 2012). Cette utopie d'une langue unique et sans reste n'est par ailleurs pas sans effet sur le fond. Conçue «sur le modèle d'un code standardisé dont les usages seraient de plus en plus normalisés», une novlangue «ne s'enrichirait plus que par l'adjonction de vocabulaires techniques univoques» (Ost, 2009, p. 364), Synonyme de repli sur un langage appauvri, cette langue peut aussi «rendre impossible tout autre mode de pensée» (Ost, 2009, p. 97). On n'en est sans doute pas si loin avec le DSM.

La troisième caractéristique de ce type d'approche descriptive est que l'adoption du DSM traduit, depuis le DSM-IV, un choix organique qui infléchit sa dimension athéorique et médicalise sensiblement la question des troubles mentaux. L'avant-propos de la traduction française du DSM-IV est sans équivoque à cet égard: «Sur le plan conceptuel, le DSM-IV mentionne la volonté de se libérer du dualisme corps-esprit et de ré-envisager sous un jour nouveau les relations entre troubles mentaux et affections médicales générales» (APA, 2002, avant-propos). Dans l'introduction au DSM-IV-TR, les auteurs insistent: «Bien que ce volume ait pour titre Manuel diagnostique et statistiques des troubles mentaux, le terme de trouble mental implique malencontreusement une distinction entre les troubles «mentaux» et les troubles «physiques», ce qui est un anachronisme réducteur du dualisme esprit/corps». Il n'existe donc "pas de différence fondamentale entre trouble mental et trouble physique» (APA, 2002, XXXV, XL) et rien n'empêche de traiter le trouble mental sur le modèle d'une médecine somatique. Cette évolution vers un modèle médical n'est pas insignifiante, dès lors que la médecine somatique est aujourd'hui pénétrée par le modèle de l'Evidence-Based Medicine (EBM) (Straus, Richardson, Glasziou et Haynes, 2006). Soucieuse de déterminer le meilleur traitement possible pour un patient donné à un moment déterminé, l'EBM propose une stratégie d'optimisation des chances de traitement qui fonctionne sur une logique probabiliste et repose sur des indicateurs statistiques obtenus en croisant diverses méthodes. Or, souligne Briffault (2013), "si l'on adopte ce modèle médical des troubles mentaux, il n'y a aucune difficulté à passer de l'EBM à l'EBBM» et de prévoir des traitements standardisés pour les troubles mentaux (p. 30-31). Cela suppose néanmoins l'adoption d'une conception du trouble comme élément visible isolé de la personne, la définition de troubles les plus précis possible, l'adoption «d'ingrédients thérapeutiques spécifiques auxquels sont associés une efficacité spécifique sur un mécanisme spécifique» (Briffault, 2013, p. 31). Les affini- 
tés électives entre logique DSM et EBMM semblent évidentes et reposent sur un postulat commun: le traitement d'un trouble mental par sa face visible, détachée de l'univers de sens dans lequel il s'inscrit pour un sujet ou l'adoption d'une «science de la surface» (Adam, 2012, p. 164) qui n'arrête de rajouter en périphérie ce qu'elle perd en profondeur.

\section{Conclusion}

Aujourd'hui, deux types de rationalité qui ont chacune leur généalogie s'affrontent dans un champ de la santé mentale aux contours fluctuants. La première fait appel à une raison scientifique et médicale. Centrée sur des troubles isolés du sujet, elle favorise une approche technique de troubles mentaux identifiés à des troubles physiques. Elle prend appui sur une démarche consensuelle, des définitions et des classifications en principe neutres et objectives, une science qui se veut dégagée des rapports sociaux, indépendante des relations de pouvoir, indifférente à la question du sens. L'approche descriptive qu'incarne le DSM depuis sa troisième version de 1980 est emblématique de cette démarche, qu'on retrouve par ailleurs aussi dans les approches cognitivo-comportementales dont le souci «scientifique» repose sur un imaginaire largement identique $^{11}$. Cette approche, qui postule sur le modèle du diagnostic médical un effacement de la subjectivité au profit d'une quête de signes objectifs, est largement dominante aujourd'hui. Sans doute parce qu'elle permet à la psychiatrie de se redonner une légitimité ancrée dans le discours de la science, mais aussi parce qu'elle répond particulièrement bien aux impératifs d'une rationalité managériale, axée plus sur la gestion des comportements socialement problématiques que sur la compréhension de leur signification pour un sujet singulier. D'un point de vue gestionnaire, la logique de système qu'incarne le DSM ou les autres échelles d'évaluation, le principe classificatoire, la réduction des individus à leurs variables mesurables et la valorisation de réponses protocolaires s'avèrent en effet séduisants. Parée des vertus de la démarche scientifique, cette logique permet de fonder en toute objectivité une norme de référence - la santé mentale - dont les fluctuations sont présentées comme le signe des avancées de la science et de définir

11. En France, le rapport de l'Inserm sur le Trouble des conduites chez l'enfant et l'adolescent, rédigé par des experts cognitivo-comportementalistes, est éclairant à cet égard (Inserm, 2005). Pour une critique de ce rapport, voir Cartuyvels (2009). 
en miroir des troubles spécifiques, conçus comme handicap, déficit ou risque futur de dysfonctionnement purement personnels. Elle permet de développer des diagnostics standardisés, selon des catégories existantes et de proposer l'application automatique de réponses correctrices ou adaptatives. Son intérêt n'est pas de comprendre ou d'aider un sujet à comprendre, mais bien de le faire fonctionner, au meilleur coût, ici et maintenant. La question du sens congédiée, c'est aussi le lien entre clinique et politique qui est évacué: une rationalité médicale mise au service de la gestion se transforme en outil de technologie sociale et a pour effet la dépolitisation des enjeux sociaux de la maladie mentale. Dans le champ de la déviance, l'imaginaire du risque qui tend à prendre la place de celui de la dangerosité (Castel, 1983) traduit le même souci de fonctionnalité. Ici aussi, échelles de risques objectifs, répondant aux «standards scientifiques internationaux les plus élevés» (Vandevelde et al., 2011, p. 74-75), sont appliquées à des sujets dont l'histoire de vie et le récit sont évacués au profit d'indicateurs prédictifs objectifs, qu'ils soient statiques ou dynamiques (Maurutto et Hannah-Moffat, 2006). Adoptant une perspective actuarielle, «la préoccupation qui ressort de cet ensemble est de permettre, sur les auteurs d'infraction, des jugements fiables qui reposent sur des risques standardisés constitués en check list en vue d'établir des guides que le juge pourrait suivre et qui détermineraient ses interventions et ses décisions» (Debuyst, 2006, p. 259). Si l'on remplace jugement par diagnostic et décision par traitement, on s'aperçoit que l'imaginaire du DSM n'est pas très loin.

En face, une autre démarche, incarnée par la psychanalyse et les thérapies d'inspiration analytique, qui prend appui sur une tradition herméneutique et une conception de la maladie mentale comme psychopathologie remontant à Hegel et à Pinel. Le pari fondateur est tout autre: il est qu'il existe toujours un sujet et une histoire derrière la souffrance qui s'exprime dans un dire ou dans un acte posant problème à son auteur. La question du sens est donc centrale et ce sens n'est pas formalisable à travers des lois ou des catégories préconstruites. Le sens est transfrontière, il s'inscrit dans une histoire identitaire qui est celle, toujours singulière, du sujet. Tant le diagnostic que le traitement ne se conçoivent dès lors pas sans la parole de ce dernier. Parce que, comme le soulignait Ricœur (1994), «la douleur n'est pas la souffrance» et que si la première peut se satisfaire d'une approche technique et médicale, la seconde renvoie «à des affects ouverts sur la réflexivité, le langage, le rapport à soi, à autrui, au sens» (p. 58-59). Ici, corps et esprit sont 
indissociablement liés, le symptôme organique renvoyant à un univers de sens à déchiffrer.

Contrairement à la première, cette approche mentaliste n'a pas le vent en poupe. En Europe francophone, elle ferait plutôt de la résistance, encore que sa disqualification, notamment universitaire, pourrait avoir comme effet d'en assurer le retour clandestin. Pourquoi ce discrédit? Au-delà de divers reproches qui ont pu lui être adressés (querelles d'écoles, langage jargonnant, etc.), un élément joue sans doute un rôle déterminant: privilégiant la question du sens, une telle approche, si elle n'échappe pas toujours aux dérives normatives, répond beaucoup moins bien aux standards orthopédiques du managérialisme contemporain. De ce point de vue, la démarche herméneutique, telle que promue par la psychanalyse, a sans doute une portée émancipatoire qui est aussi potentiellement subversive. Loin de proposer de suturer à tout prix le trouble à des fins d'apaisement, elle propose au contraire de faire parler le symptôme, dans un travail de dévoilement qui renvoie le sujet à sa propre liberté et responsabilité. À la différence d'une approche médicalisante de la santé mentale, elle n'entend pas inféoder un sujet déficitaire à une normativité externe dont le médecin ou le psychothérapeute serait le gardien. Elle vise au contraire à faire appel à une subjectivité réflexive, appelée à marquer sa liberté par une démarche de création et d'auto-institution dans un scénario jamais joué d'avance (Castoriadis, 1990, p. 183).

\section{Références}

Advenier, F. et Kapsambelis, V. (2013). Le diagnostic psychiatrique à l'ère industrielle. Topiques, 123, 7-22.

American Psychiatric Association. (1983). DSM-III. Manuel diagnostique et statistique des troubles mentaux. Paris, France: Masson.

American Psychiatric Association. (2002). DSM-IV-TR. Manuel diagnostique et statistique des troubles mentaux. Paris, France: Elsevier-Masson.

Aragona, M. (2009). The concept of mental disorder and the DSM-V. Dial Phil Ment Neuro Sci, 1, 1-14.

Bauherz, G., Kaminski, D. et Zombeck, S. (1992). Le bon usage, prévention du sida à l'adresse des usagers de drogues. Bruxelles, Belgique: Agence de prévention du sida de la Communauté française.

Bourdieu, P. (1993). La misère du monde. Paris, France: Seuil.

Callon, M., Lascoumes, P. et Barthes, Y. (2001). Agir dans un monde incertain. Essai sur la démocratie technique. Paris, France: Seuil. 
Canguilhem, C. (1968). Psychologie. Dans C. Canguilhem, Etudes d'histoire et de philosophie des sciences (p. 365-381). Paris, France: Vrin.

Cartuyvels, Y. (2004). Généralisation de la psychose? Dans Collectif d'auteurs, Fonction et usages de l'institution. Actes de la journée d'études du 30 novembre 2002: Enaden: 20 ans (p. 129-139). Bruxelles, Belgique.

Cartuyvels, Y. (2009). Troubles de conduite et déviance: des amours en eaux troubles. Revue de Droit Pénal et de Criminologie, 1, 32-61.

Cartuyvels, Y. (2012). La sortie du circuit de défense sociale: évaluer le risque et encadrer la dangerosité. Dans C. Lanceleve, M. Bessin, F. Dugué, Cartuyvels, Y. et G. Cliquennois, Ce que la dangerosité fait aux pratiques. Entre soin et peine. Une comparaison France-Belgique (p. 124-175). France: Mission Droit et Justice.

Castel, R. (1983). De la dangerosité au risque. Actes de la Recherche en Sciences Sociales, 47-48, 94-123.

Castel, R. (1995). Les métamorphoses de la question sociale: une chronique du salariat, Paris, France: Fayard.

Castel, R. (2003). L'insécurité sociale. Qu’est-ce qu'être protégé? Paris, France: Seuil.

Castells, M. (1996). The Rise of the Network Society. Oxford, Royaume-Uni: Blackwell Publishers.

Castoriadis, C. (1990). Psychanalyse et politique. Dans C. Castoriadis, Le monde morcelé. Les carrefours du labyrinthe (p. 141-154). Paris, France: Seuil.

Cooper, R. (2005). Classifying Madness. A Philosophical Examination of the Diagnostic and Statistical Manual of Mental Disorders. Doordrecht, Pays-Bas: Springer.

De Coninck, F., Cartuyvels, Y, Franssen, A., Kaminski, D., Mary, P., Réa, A. et Van Campehoudt, L. (2005). Aux frontières de la Justice, aux marges du social. Gand, Belgique: Academia Press.

Debuyst, C. (2006). Dangerosité, tables de prédiction. La fausse clandestinité d'une notion. Dans Une criminologie de la tradition à l'innovation. En hommage à G. Kellens (p. 251-265). Bruxelles, Belgique: Larcier.

Demailly, L. (2011). Sociologie des troubles mentaux. Paris, France: La découverte.

Demazeux, S. (2013). Qu'est-ce que le DSM? Paris, France: Ithaques.

Demunck, J. (2004). Réseau, gouvernance, inconsistance. Quarto, 82, 51-58.

Donzelot, J. et Estebe, X. (1994). L'État animateur. Essai sur la politique de la ville. Paris, France: Esprit.

Drossart, F. (2009). Vous avez aimé la novlangue? Vous allez adorer le DSMIV. Psychiatrie Française, 2, 162-172.

Dumont, D. (2008). Vers un État social actif? Journal des Tribunaux, 6300, 133139.

Ehrenberg, A. (1991). Le culte de la performance. Paris, France: Calmann-Levy.

Ehrenberg, A. (1995). L'individu incertain. Paris, France: Calmann-Levy.

Ehrenberg, A. (2006), Malaise dans l'évaluation de la santé mentale, Esprit, $\mathrm{n}^{\circ} 5$, 89-93. 
Ehrenberg, A. (2004). Les changements de la relation normal-pathologique. A propos de la souffrance psychique et de la santé mentale. Esprit, 304, 133156.

Ehrenberg, A. et Lovelle, A. M. (2001). Pourquoi avons-nous besoin d'une réflexion sur la psychiatrie? Dans A. Ehrenberg et A. M. Lovelle (dir.), La maladie mentale en mutation. Psychiatrie et société (p. 9-39). Paris, France: Odile Jacob.

Feys, J.-L. (2009). L'anthropopsychiatrie de Jacques Schotte. Une introduction. Paris, France: Hermann.

Fineltain, L. (2011). Les classifications en psychiatrie. Bulletin de psychiatrie, 20, $1-17$.

Frances, A. (2010a). Rating scales: DSM5 Bites Off Far More Than It Can Chew. Psychiatric Times. Repéré à www.psychiatrictimes.com/articles/ rating-scales-dsm5-bites-far-more-it-can-chew

Frances, A. (2010b). Opening Pandora's Box: The 19 Worst Suggestions For DSM-5. Psychiatric Times, 11. Repéré à www.psychiatrictimes.com/display/ article/10168/1522341

Frances, A. (2010c). DSM5: Pandora Replies to Dr Frances. Psychiatric Times. Repéré à www.psychiatrictimes.com/articles/dsm5-pandora-replies-drfrances

Frances, A. (2013a, 9 mai). La psychiatrie est en dérapage incontrôlé. Le Nouvel Observateur. Repéré à http://bibliobs.nouvelobs.com/en-partenariat-avecbooks/20130329.OBS6215/allen-frances-la-psychiatrie-est-en-derapageincontrole.html

Frances, A. (2013b). Saving normal: An Insider's Revolt Againts Out-of-Control Psychiatric Diagnosis DSM 5, Big Pharma, and the Medicalisation of ordinary life. New-York, NY: William Morrow.

Frances, A. (2013c, 19 mai). We're all mad here, New York Post. Repéré à http:// nypost.com/2013/05/19/were-all-mad-here/

Freud, S. (2005). Le malaise dans la culture. Paris, France: PUF.

Garapon, A. (2008). Un nouveau modèle de justice: efficacité, acteur stratégique, sécurité. Esprit, 11, 98-122.

Genard, J.-L. et Donay, J.-Y. (2002). L'action publique en matière de santé mentale. Revue Nouvelle, 2, 70-79.

Habermas, J. (1988). Le discours philosophique de la modernité. Paris, France: Gallimard.

Institut national de la santé et de la recherche médicale [INSERM]. (2005). Les troubles de conduite chez l'enfant et l'adolescent. Paris, France: Editions de l'Inserm.

Lane, C. (2009). Comment la psychiatrie et l'industrie pharmaceutique ont médicalisé nos émotions. Paris, France: Flammarion.

Lebrun, J.-P. (1997). Un monde sans limites. Malaise dans la subjectivation. Toulouse, France: Eres.

Lebrun, J.-P. (2007). La perversion ordinaire, vivre ensemble sans autrui. Paris, France: Denoël. 
Legendre, P. (1993). Ce que nous appelons le droit: Entretien avec P. Legendre. Le Débat, 74, 107-122.

Maleval, J.-C. (2003). Limites et dangers du DSM. L'évolution Psychiatrique, 68, $39-61$.

Marquis, N. (2013). What Can I Do to Get Out of It? How self-help readers Make Use of the Language Game of Resilience. $M$. C. Journal, 5. Repéré à http://journal.media-culture.org.au/index.php/mcjournal/article/ view/693

Martens, F. (2002).Comment être fou dans les règles. Revue Nouvelle, 2, 44-48.

Martens, F. (2010). La candeur de Gagarine. Revue Nouvelle, 3, 82-89.

Maurutto, P. et Hannah-Moffat, K. (2006). Assembling risk and the restructuring of penal control. British Journal of Criminology, 46, 438-454.

Mayes, R. et Horwitz, A. (2005). DSM-III and the Revolution in the Classification of Mental Ilness. Journal of the History of the Behavioral Sciences, 3, 249-267.

Ost, F. (2009). Traduire. Défense et illustration du multilinguisme. Paris, France: Fayard.

Paris, J. et Philips, J. (2013). Making the DSM-V. Concepts and controversies. Doordrecht, Pays-Bas: Springer.

Ricoeur, P. (1994). La souffrance n'est pas la douleur. Autrement, 142, 58-69.

Schweyer, F.-X. (2006). Une profession de l'État providence, les directeurs d'hôpital. European Journal of Social Sciences, 135, 45-60.

Singy, P. et Demazeux, S. (2013, 29 mai). Du DSM_IV au DSM 5: le jour d'après. Repéré à www.slate.fr/tribune/72919/dsm-iv-dsm-5-psychiatrie

Straus, S. E., Richardson, W.S., Glasziou, P. et Haynes, R. B. (2007). Médecine fondée sur les faits - Evidence-Based Medecine. Paris, France: Elsevier-Masson.

Van Campenhoudt, L. (2012). Vaut-il toujours mieux lever les malentendus? Revue Nouvelle, 3, 111-117.

Vandevelde, S., Soyez, V., Vander Beken, T., De Smet, S., Boers, A. et Broeckaert, E. (2001). Mentally ill offenders in prison: The Belgian case. International Journal of Law and Psychiatry, 34, 71-78.

Vrancken D. (2002), Lorsque la santé mentale révèle de nouvelles formes d'action sociale, La Revue Nouvelle, 2, 38-43.

Wakefield, J. C. (1992). Disorder as Harmful Dysfunction: A Conceptual Critique of DSM-III-R's Definition of Mental Disorder. Psychological Review, 2, 232-247. 


\title{
Transformations in the Mental Health Sector: Between the Science and the Subject
}

\begin{abstract}
This article examines the historical transformations of the mental health sector, mostly from a European, French speaking point of view, and in particular France and Belgium. It describes the institutional and material mutations of the mental health sector and, more specifically, the rise of the DSM, its ideology and social consequences. Ultimately, these transformations can be considered in terms of opposing orientations currently dominating the field: On the one hand, the scientific approach that would ideally rid itself of the human subject and, on the other hand, an approach that holds subjectivity as essential and irreducible.
\end{abstract}

KEYWORDS - Mental health, historical transformations, evolution, DSM, critique.

\section{Transformaciones de la salud mental: Entre la ciencia y el sujeto}

RESUMEN - El artículo estudia las transformaciones históricas en el área de la salud mental, principalmente a través de las resonancias contemporáneas en la Europa francófona y principalmente en Francia y en Bélgica. Describe las mutaciones desde un punto de vista institucional y material, examinando, particularmente y de forma crítica, el alcance del DSM, el imaginario que vehicula y sus consecuencias sociales. In fine, estas transformaciones pueden ser comprendidas entre una aproximación científica, que soñaría con deshacerse del sujeto humano y otra que lo considera esencial e irreductible.

PALABRAS CLAVE • Salud mental, transformaciones históricas, evolución, DSM, crítica. 